Revista Tecné, Episteme y Didaxis: TED. Año 2014, Número

Extraordinario. ISSN Impreso: 0121-3814, ISSN web: 2323-0126

Memorias, Sexto Congreso Internacional sobre Formación de

Profesores de Ciencias. 08 al 10 de octubre de 2014, Bogotá

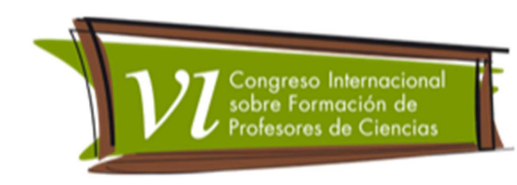

\title{
Articulación de las Cuestiones Sociocientíficas al Currículo de Ciencias: Aportes y Limitaciones para la Formación de Profesores en la Interfaz Universidad-Escuela
}

Arias Ingrid Ximena ${ }^{1}$

Categoría 2. Trabajo de investigación (en proceso).

\section{Resumen}

Esta ponencia, se enmarca en torno al desarrollo de un proyecto de investigación que se viene adelantando en la Facultad de Ciencia y Tecnología de la Universidad Pedagógica Nacional, cuya finalidad es analizar, qué dificultades presenta el pequeño grupo de investigación (PGI) del IED. Fabio Lozano Simonelli, durante la articulación de CSC al currículo oficial de ciencias y qué aportes tiene la articulación de CSC en la formación de profesores. Para tal fin, este documento centra la atención en los aspectos teóricos y metodológicos de dicha investigación, relacionados con CTSA, CSC y formación de profesores, así como la elaboración de instrumentos y caracterización de los participantes.

\section{Palabras Clave}

Enfoque CTSA, Enseñanza de las Ciencias, interdisciplinaridad.

\section{Objetivos}

\section{Objetivo General}

Analizar las dificultades presentadas durante la articulación de CSC en el currículo de ciencias del IED Fabio Lozano Simonelli, a través del acompañamiento del PGI de esta institución.

\section{Objetivos Específicos}

Estudiar los aportes de la articulación de CSC en un programa de formación permanente de profesores situado en la interfaz universidad-escuela.

\footnotetext{
1 Universidad Pedagógica Nacional, mena.45@hotmail.com
} 
Revista Tecné, Episteme y Didaxis: TED. Año 2014, Número Extraordinario. ISSN Impreso: 0121-3814, ISSN web: 2323-0126

Memorias, Sexto Congreso Internacional sobre Formación de Profesores de Ciencias. 08 al 10 de octubre de 2014, Bogotá

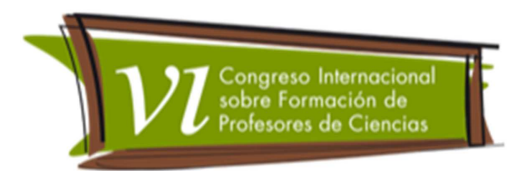

\section{Marco teórico}

La enseñanza de las ciencias lineal, precisa, incuestionable y la existencia de estructuras rígidas, propuestas en muchas ocasiones, por sujetos ajenos a las necesidades que se vivencian en las aulas de clase, no solo han hecho que los profesores pierdan su participación y autonomía ante la consolidación de dichos lineamientos, sino que dada la incoherencia entre lo teórico, lo práctico y lo que se vive en la sociedad actual, ha generado en los estudiantes actitudes negativas, falta de interés y motivación hacia el aprendizaje de las ciencias. En ese sentido, se presentan las cuestiones sociocientíficas como situaciones sustentadas desde una educación científica para la acción social, que permiten renovar el currículo oficial y mejorar el proceso de enseñanza y aprendizaje de las ciencias.

Cuestiones Sociocientíficas (CSC) desde el enfoque, Ciencia, Tecnología y Sociedad y Ambiente (CTSA):

El enfoque Ciencia, Tecnología, Sociedad y Ambiente (CTSA) en la enseñanza de las ciencias surge en la década de 1970 como un movimiento de renovación curricular, que reclamaba una enseñanza crítica en oposición al cientificismo y tradicionalismo predominante en la educación de la época (Martinez, Parga, \& Gomez, 2012). En contraste, la incorporación de este movimiento en la escuela, pretendía forjar una educación científica más humanística, donde la enseñanza estuviera destinada a promover una ciencia escolar de mayor validez y utilidad para los estudiantes, que como ciudadanos responsables, tenían que prepararse para tomar decisiones respecto a cuestiones de la vida real, íntimamente relacionadas con aspectos científicos y tecnológicos.

Sin embargo, a pesar de que la finalidad educativa para el movimiento CTSA es clara y da sentido al proceso de aprendizaje, este ha enfrentado varias dificultades para concretarse en el currículo (Martinez, Parga, \& Gomez, 2012). Lo cual, ha derivado la búsqueda de nuevas alternativas que logren conquistar los retos propuestos por este enfoque, entre estas, se encuentran los trabajos relacionados con situaciones controvertidas denominadas como CSC, de hecho Martínez (2010) en sus investigaciones, afirma que la articulación de CSC en el aula son una forma concreta de incorporar las interacciones CTSA en las prácticas del profesorado. 
Revista Tecné, Episteme y Didaxis: TED. Año 2014, Número Extraordinario. ISSN Impreso: 0121-3814, ISSN web: 2323-0126

Memorias, Sexto Congreso Internacional sobre Formación de Profesores de Ciencias. 08 al 10 de octubre de 2014, Bogotá

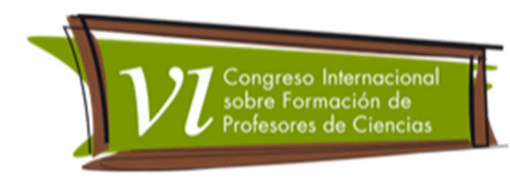

\section{Características de las Cuestiones Sociocientíficas (CSC)}

Formar ciudadanos capaces de intervenir y participar en las cuestiones generales y próximas que se presentan en la cotidianidad, es un asunto urgente que se hace necesario cada día para la toma de decisiones concernientes a aspectos científicos y tecnológicos contemporáneos. Razón por la cual, las CSC pretenden que el estudiante, preste atención en el ejercicio de la ciudadanía y se prepare para la participación en discusiones relacionadas con asuntos tecnocientíficos (Acevedo, y otros, 2005).

En ese orden de ideas, Pinzon, Salazar \& Martínez (2013) consideran que las CSC son asuntos propios de un lugar in situ, los cuales al encontrarse de forma explícita en la naturaleza sociocultural del conocimiento científico, están sujetas a debates, polémicas, controversias y diversas soluciones desde diferentes perspectivas. Por lo cual, poseen una base científica que a menudo hace parte de investigaciones de frontera, involucran la formación de opiniones, son frecuentemente divulgadas por los medios de comunicación, pueden tener evidencias científicas o sociales incompletas y se pueden abordar las dimensiones locales, nacionales y mundiales.

\section{Formación de profesores en la interfaz universidad - escuela}

El interés por investigar sobre la formación inicial y continuada de profesores de ciencias, se generó durante el siglo XX, a finales de los años 80 inicios de los 90 (Martínez \& Carvalho, 2013), a fin de mejorar, la calidad de la enseñanza de las ciencias. Sin embargo, a pesar de las múltiples investigaciones que se han llevado a cabo en torno al tema, estudios recientes cuestionan aún, la ausencia de discusión por parte de profesores de ciencias, respecto a las implicaciones sociales, culturales y ambientales del emprendimiento científico y tecnológico. Pasividad que en últimas, ha contribuido a fortalecer la visión tradicional de la Enseñanza de las Ciencias (Martinez, Parga, \& Gomez, 2012).

Frente a lo expuesto, De Carvalho (2005 citado en Martínez \& Carvalho, 2013) afirma, que los proyectos no puede ser desarrollados solamente en la escuela o en la universidad, por el contrario, estos, deben ser uno de los medios de interlocución entre universidad y escuela. Para que mediante el consenso de las diferencias que existen en cada campo y el trabajo colectivo, no solo se contribuya en los intereses que posee cada profesor en relación con su profesión, sino que adicionalmente mejore la enseñanza de las ciencias. 
Revista Tecné, Episteme y Didaxis: TED. Año 2014, Número Extraordinario. ISSN Impreso: 0121-3814, ISSN web: 2323-0126

Memorias, Sexto Congreso Internacional sobre Formación de Profesores de Ciencias. 08 al 10 de octubre de 2014, Bogotá

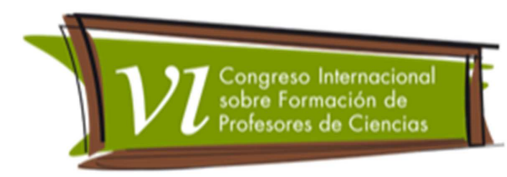

\section{Aportes de la implementación de las CSC en la formación de profesores}

Martínez (2010), afirma que el abordaje de las CSC en la escuela, aporta en la formación del profesor como investigador de su propia práctica y posibilita la generación y/o fortalecimiento de la autonomía docente. Puesto que, dada la interdisciplinariedad de estas situaciones controvertidas, no solo pueden ser abordadas por los profesores de ciencias, sino que análogamente, pueden ser planteadas por profesores de diferentes áreas y estar acompañadas por profesores universitarios durante su diseño e implementación (Martinez, Parga, \& Gomez, 2012).

No obstante, a pesar de que son muy pocos los profesores de escuela y de universidad comprometidos con la enseñanza y el aprendizaje, basado en la investigación escolar, es necesario la construcción de nuevos espacios formativos, donde por medio de la participación de profesores en servicio y profesores universitarios se estimulen y dinamicen la elaboración de trabajos e investigaciones colectivas.

\section{Metodología}

Este trabajo investigativo, se orienta bajo las características de un diseño de tipo cualitativo, estudio de caso. El cual, tiene como objeto de estudio el pequeño grupo de investigación (PGI) conformado en el IED Fabio Lozano Simonelli, ubicado en el Barrio la Fiscala, a fin de analizar las dificultades presentadas durante la implementación de las CSC al currículo de ciencias. Por esto, las herramientas diseñadas para la recolección de información durante la fase de caracterización fueron: dos observaciones participantes durante la consolidación de la CSC, relatorías y un cuestionario inicial, que buscaba indagar las percepciones de los docentes implicados sobre: investigación, relaciones ciencia, tecnología, sociedad y ambiente (CTSA)y CSC.

\section{Resultados}

En relación con la población escogida, se aclara que esta investigación, se enmarca dentro del proyecto de investigación que ha venido adelantando el grupo Alternaciencias: Programa Colombo-Brasileño de Formación de Profesores de Ciencias en La Interfaz Universidad-Escuela Col-Upn-537-12, espacio en el que se conforman siete PGI, a fin de discutir asuntos relacionados con la mejora de los procesos de enseñanza y aprendizaje. Partiendo de lo mencionado, para elegir uno de los siete PGI, se tuvieron en cuenta, aspectos como: la participación de profesores que se 
Revista Tecné, Episteme y Didaxis: TED. Año 2014, Número Extraordinario. ISSN Impreso: 0121-3814, ISSN web: 2323-0126

Memorias, Sexto Congreso Internacional sobre Formación de Profesores de Ciencias. 08 al 10 de octubre de 2014, Bogotá

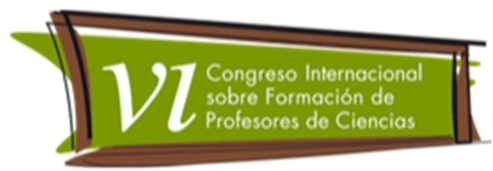

desempeñaran en diversas áreas del conocimiento para poder dar cuenta de la interdisciplinariedad de las CSC. Que algunos profesores del PGI estuvieran vinculados a estudios de postgrado o a encuentros propuestos por el grupo Alternaciencias, con el objetivo de generar trabajo colectivo desde la interfaz universidad - escuela y finalmente la disposición y disponibilidad de los profesores y la institución para llevar a cabo el trabajo de investigación.

En ese sentido, los resultados conseguidos en el instrumento inicial manifestaron percepciones iniciales de los diez docentes del IED. Fabio Lozano Simonelli sobre asuntos relacionados con: investigación, CSC y CTSA. Es así, como el 60\% consideran que la investigación en la escuela es una oportunidad para reflexionar y mejorar la práctica docente, de hecho, una de las respuestas encontradas asume la investigación como "la posibilidad de reflexionar acerca de las situaciones que van a pareciendo día a día en la práctica, permitiendo indagar de una manera más profunda y mejorar como docente". El 40\% restante, opinan que investigar significa aproximarse al conocimiento para encontrar información que complemente las clases, resolver inquietudes de forma individual o colectiva a los estudiantes, o por el contrario, la oportunidad para profundizar en temas no sugeridos por el currículo.

Respecto a CTSA, el $80 \%$ de la población afirma no haber tenido algún encuentro donde se aluda a las relaciones entre ciencia, tecnología, sociedad y ambiente. Solamente el $20 \%$ de la población manifiestan que en proyectos de investigación de maestría y el proyecto actual en el que se encuentran vinculados, el trabajo con el PGI y por supuesto el planteamiento de CSC ha posibilitado vivenciar la relación existente entre CTSA, uno de las apreciaciones manifiesta que: "Si, el proyecto de investigación de la maestría, en el cual se planteó una CSC relacionada con los polímeros, frente a esta se analizó las implicaciones sociales, tecnológicas y científicas, fue bastante interesante promover este tipo de experiencia en el aula, ya que se refleja el interés de los estudiantes, como ellos desarrollan habilidades como la argumentación y pensamiento crítico y por supuesto en el trabajo con el PGl el cual está vinculado al proyecto de investigación donde participo actualmente".

En cuanto a las concepciones sobre CSC, el 80\% de la población manifiestan que han escuchado sobre qué son y que caracteriza a las CSC, entre las afirmaciones se encuentra: "Si, las CSC son dilemas sociales que tienen una base científica, pero que a su vez se relaciona con otros campos del conocimiento como el social, económico, político ambiental; generalmente son conocimientos de frontera, entre sus características se encuentra que son frecuentemente divulgadas por los medios de 
Revista Tecné, Episteme y Didaxis: TED. Año 2014, Número Extraordinario. ISSN Impreso: 0121-3814, ISSN web: 2323-0126

Memorias, Sexto Congreso Internacional sobre Formación de Profesores de Ciencias. 08 al 10 de octubre de 2014, Bogotá

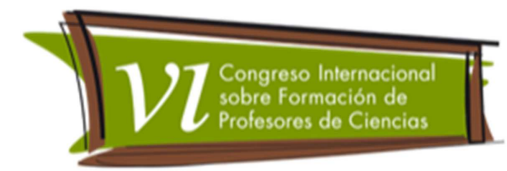

comunicación, presentan diversidad de opiniones, puede tener evidencias científicas y sociales incompletas y se pueden abordar dimensiones locales, nacionales $y$ mundiales", el $20 \%$ de la población restante no tiene conocimiento acerca de lo que son o qué caracterizan a las CSC.

\section{Conclusiones}

A la luz de los resultados obtenidos y los referentes teóricos, se concluye, que a pesar de que gran parte de la población percibe la investigación escolar como la oportunidad de mejorar profesionalmente, algunos manifiestan que la investigación es una actividad complementaria del docente que le permite responder inquietudes al estudiante y profundizar en temas no sugeridos en el currículo.

Con relación al conocimiento de los profesores sobre CTSA, se identificó que aunque la mayoría de los docentes están vinculados a un proyecto de investigación íntimamente relacionado con las interacciones entre ciencia, tecnología, sociedad y ambiente, solo el $20 \%$ reconoce que lo han trabajado, el resto de los docentes poseen visiones distorsionadas de lo que es CTSA en la enseñanza. Finalmente, pudo identificarse que el $80 \%$ de la población tiene conocimiento sobre algunas de las características de las CSC, lo cual facilita la identificación y consolidación de la CSC a trabajar en el PGI, según las necesidades halladas en los estudiantes.

\section{Referencias bibliográficas}

Acevedo, J., Vásquez, Á., Martín, M., Oliva, J., Acevedo, P., Paixao, M. F., y otros. (2005). Naturaleza De La Ciencia Y Educación Científica Para La Participación Ciudadana. Una Revisión Crítica. Revista Eureka sobre Enseñanza y Divulgación de las Ciencias, 121 - 140.

Martínez, L. (2010). A abordagem de questões sociocientíficas na formação continuada de professores de Ciências: contribuições e dificuldades. Tese (Doutorado). Faculdade de Ciências da Universidade Estadual Paulista, Bauru. Disponible en:http://www2.fc.unesp.br/BibliotecaVirtual/DetalhaDocumentoAction.do?idDo cumento=325\# . Acesso el: 10 de abril de 2014.

Martínez, L. F., Parga, D. L., \& Gomez, D. L. (2012). Cuestiones sociocientíficas en la Formación de Profesores de Ciencias. Asociación Colombiana para la investigación en Educación en Ciencias y Tecnología EDUCyT, 139-151. 
Revista Tecné, Episteme y Didaxis: TED. Año 2014, Número

Extraordinario. ISSN Impreso: 0121-3814, ISSN web: 2323-0126

Memorias, Sexto Congreso Internacional sobre Formación de

Profesores de Ciencias. 08 al 10 de octubre de 2014, Bogotá

Martínez, L., \& Carvalho, W. (2013). Programa Colombo-Brasileño De Formación De Profesores De Ciencias En La Interfaz Universidad-Escuela Col-Upn-531-12. Bogotá: Capes/Colciencias.

Pinzón, Y., Salazar, L., \& Martínez, L. (2013). Características de Las Cuestiones Sociocientíficas en la enseñanza de las Ciencias. Documento del Programa Colombo-Brasileño de Formación de Profesores de Ciencias en la Interfaz. 